\title{
Hair analysis of cortisol levels in adrenal insufficiency
}

\author{
Christine Ibrahim MD, Stan Van Uum MD PhD
}

Competing interests: None declared.

This article has been peer reviewed.

Affiliations: Division of General Internal Medicine (Ibrahim), Divisions of

Endocrinology and Metabolism and Clinical Pharmacology (Van Uum), Department of Medicine, Schulich School of Medicine and Dentistry, London, Ont.

Correspondence to: Stan Van Uum, stan.vanuum @ sjhc.london.on.ca

\section{CMAJ 2014.DOI:10.1503} /cmaj.140407
1 19-year-old woman was admitted to hospital with nausea, vomiting, urinary tract infection and severe hypotension that necessitated a five-day stay in the intensive care unit (ICU). A few weeks later, she was readmitted to hospital with a respiratory infection. Her serum sodium level was 130 (normal 135-145) $\mathrm{mmol} / \mathrm{L}$, potassium level 6.1 (normal 3.5-5) $\mathrm{mmol} / \mathrm{L}$, cortisol level less than 11 (normal 120-384) nmol/L and adrenocorticotropic hormone level greater than 278 (normal 0.0 10.1) $\mathrm{pmol} / \mathrm{L}$; these results were diagnostic of acute adrenal insufficiency crisis. Aldosterone and renin levels (expected to be low and high, respectively) were not measured. The patient was prescribed hydrocortisone and fludrocortisone. Her detailed history suggested that the adrenal insufficiency had developed much earlier, with a three- to four-year history of unintended weight loss $(18 \mathrm{~kg})$, progressive weak-

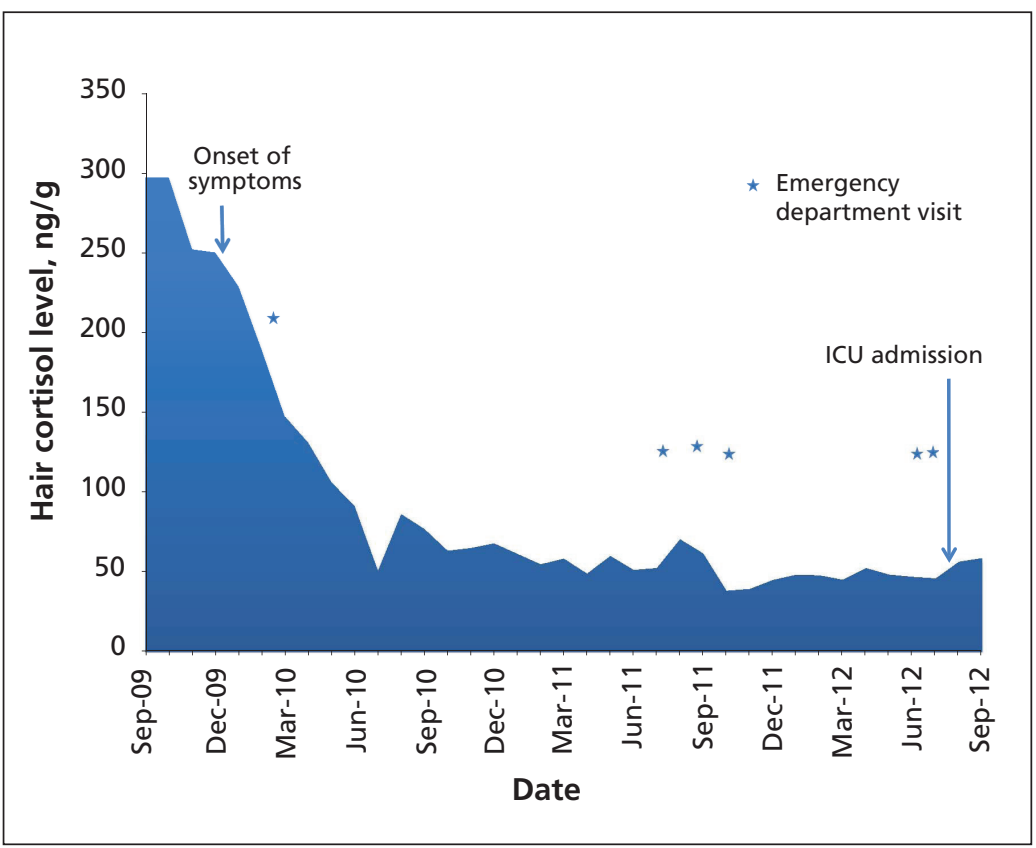

Figure 1: Cortisol levels in a sample of hair analyzed in 1-cm sections from a 19-year-old woman with chronic adrenal insufficiency. ICU = intensive care unit. ness, fatigability, tanned skin and episodes of orthostatic presyncope. Her health status was stable between episodes of illness but deteriorated rapidly when she was ill.

The patient provided a hair sample of about $36 \mathrm{~cm}$ in length. Analysis of 1-cm sections of the sample showed the retrospective development of impaired cortisol production (Figure 1). The decline in cortisol production started almost three years before her admission to the ICU and coincided with the onset of symptoms of adrenal insufficiency.

Because hair grows at a rate of about $1 \mathrm{~cm}$ per month, longer hair samples can reflect cortisol production over several months to years. ${ }^{1}$ In patients with Cushing syndrome, measurement of cortisol levels in hair can reflect changes in systemic levels of cortisol., ${ }^{2,3}$ It is not known whether adrenal insufficiency changes the rate of hair growth. Measurement of cortisol levels in hair may provide insight into the natural history of adrenal insufficency. Moreover, in patients with an unconfirmed diagnosis who have already started glucocorticoid treatment, it may provide information on cortisol levels before the treatment began. Patients need to be able to provide samples of scalp hair that are at least $1 \mathrm{~cm}$ in length, and use of glucocorticoids and hydrocortisone cream must be determined.

Hair analysis of historical cortisol levels is a promising tool for use in clinical practice but requires further study.

\section{References}

1. Gow R, Koren G, Rieder M, et al. Hair cortisol content in patients with adrenal insufficiency on hydrocortisone replacement therapy. Clin Endocrinol (Oxf) 2011;74:687-93.

2. Thomson S, Koren G, Fraser LA, et al. Hair analysis provides a historical record of cortisol levels in Cushing's syndrome. Exp Clin Endocrinol Diabetes 2010;118:133-8.

3. Manenschijn L, Koper JW, van den Akker EL, et al. A novel tool in the diagnosis an follow-up of (cyclic) Cushing's syndrome: measurement of long-term cortisol in scalp hair. J Clin Endocrinol Metab 2012;97:E1836-43.

Acknowledgments: The authors thank Evan Russell for the analysis of cortisol in the hair sample and the patient who provided the sample and agreed to share this clinical vignette. 